\title{
Preface for minimally invasive surgery for lung cancer
}

With advancements in computed tomography screening program for early lung cancer, small lung nodules have been increasingly detected. Recently, uniportal and nonintubated VATS for major lung resections has become a revolution in the treatment of lung cancer. These novel minimally invasive thoracic surgery techniques for small, impalpable early lung cancer is challenging for physicians, and preoperative localization techniques have become even more important than they were in the past. This focused issue aims to provide a comprehensive overview on a variety of aspects related to recent update of minimally invasive surgery for lung cancer, as well as on image-guided preoperative localization techniques.

\section{Acknowledgements}

None.

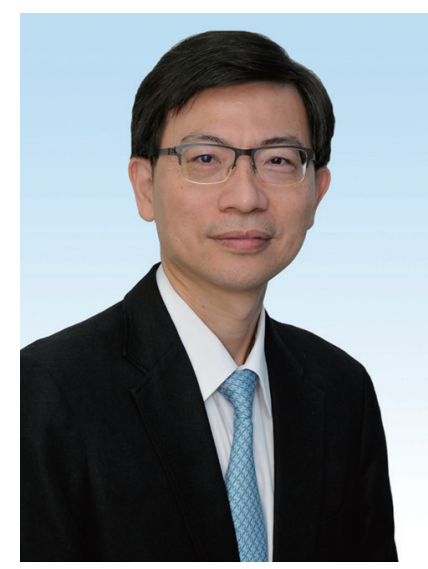

Jin Shing Chen

Jin Shing Chen ${ }^{1,2,3}$

${ }^{1}$ Professor, Department of Surgery, National Taiwan University, Taipei, Taiwan;

${ }^{2}$ Chief, Division of Thoracic Surgery, Department of Surgery,

${ }^{3}$ Chief, Division of Experimental Surgery, Department of Surgery, National Taizwan University Hospital,

Taipei, Taiwan.(Email: chenjs@ntu.edu.tw)

doi: 10.21037 /jovs.2018.05.10

Conflicts of Interest: The author has no conflicts of interest to declare.

View this article at: http://dx.doi.org/10.21037/jovs.2018.05.10

doi: 10.21037 /jovs.2018.05.10

Cite this article as: Chen JS. Preface for minimally invasive surgery for lung cancer. J Vis Surg 2018;4:91. 